\title{
The Development of Wireless Sensor System for Pressure and Temperature Signals Monitoring
}

\author{
Essa Jafer \\ University of Limerick \\ College of Informatics and Electronics \\ Limerick, Ireland \\ 353- (0)61-202267 \\ Essa.jafer@ul.ie
}

\author{
Khalil Arshak \\ University of Limerick \\ College of Informatics and Electronics \\ Limerick, Ireland \\ 353- (0)61-202267 \\ Khalil.Arshak@ul.ie
}

\begin{abstract}
In this work, a design of wireless system for multi-sensors monitoring has been presented. The main purpose of this research is to develop an efficient telemetry system for measuring water pressure and temperature signals. The system reads data from two types of sensors, resistive and capacitive. Anderson circuit configuration has been used for the resistive sensor, since it offers linear like behavior. The capacitive interface is based on capacitance-frequency-voltage conversion that uses phase lock loop (PLL) to convert the frequency to voltage. A developed pressure sensors based on polyvinylidene fluoride (PVDF) was used to evaluate the performance of the capacitive interface. Two mechanisms have been adopted to reduce the overall power consumption. These are power scheduling and wakeup circuit for the capacitive interface. The first one is implemented fully by the micro-controller unit (MCU) in order to switch on/off all the system units. A wakeup circuit has been designed to interrupt the MCU when it is in the sleep mode and the capacitive samples are changing significantly. A platform for wireless sensor network (WSN) has been developed so that the receiver side can communicate with more than one sensor node.
\end{abstract}

\section{Categories and Subject Descriptors}

B.4.1 [Input/output and Data Communication]: Data Communication Devices - receivers, transmitters.

\section{General Terms}

Management, Measurement, Performance, Design, Experimentation, Verification.

\section{Keywords}

Wireless sensors, System monitoring, Pressure, Temperature.

\section{INTRODUCTION}

There is a considerable interest in the development of multisensor micro-systems for use in the implanted [1], ingestible [2] and remote environmental monitoring [3]. Building a complete sensor micro-system involves several challenges as these designs

Permission to make digital or hard copies of all or part of this work for personal or classroom use is granted without fee provided that copies are not made or distributed for profit or commercial advantage and that copies bear this notice and the full citation on the first page. To copy otherwise, to republish, to post on servers or to redistribute to lists, requires prior specific permission and/or a fee. AMBI-SYS 2008, February 11-13, Quebec, Canada

Copyright $\odot 2008$ ICST 978-963-9799-16-5

DOI 10.4108/ICST.AMBISYS2008.2884 include not only a union of the analog and digital circuit domains, but also the magnetic, mechanical, biological, chemical, or electrical domains. Such systems must exhibit low-cost production, robustness of use and real-time data processing.

In the field of environment monitoring, a number of systems have been developed and applied. A wireless sensor module for recording different ambient conditions like temperature and humidity is presented in [4]. Such system uses high rate RF transceiver and field programmable gate array (FPGA) to implement digital signal processing (DSP) tasks. Another wireless remote environment monitoring system is presented in [5], where the MCU is operating in sleep and wakeup modes to reduce power consumption. In [6], a system that utilizes inductive power and data transfer through a backscatter-modulated carrier and a transducer interface that monitors its environment through embedded capacitive transducers is introduced.

Measuring the water pressure and temperature under different conditions is still important to be determined. For examples, many systems have been presented in the past to sense the water pressure under the sea and the ground $[7,8]$. Recently wireless technology became attractive for remote monitoring where the data recorded by different sensors can be recorded and sent to a base station for analyzing 9]. Due to this, we developed a new wireless smart system for localized monitoring of both temperature and pressure in real time by means of centralized wireless receiver. The work in this paper focused mainly on the development of pressure sensors and the design of an efficient power management for the transmitter side of the system.

\section{SYSTEM ARCHITECTURE}

Figure.1 shows the system overview diagram. The word mote is used here as a short term for describing the wireless remote sensor. Based on the commands sent by the base station, the mote is configured to be for either resistive or capacitive measurements. The output samples from the signal conditioning circuit will be processed and buffered by a microcontroller before the transmitter becomes ready. The data will be sent to the Base Station over 433 $\mathrm{MHz}$ channel using Frequency Shift Keying (FSK) modulation type. The mote also has the ability to power off the appropriate 
conditioning interfaces if they are not being used or between the sampling times of the two analog signals to save power [10]. The bi-directional wireless communication will be responsible to direct the mote system by sending suitable commands and on the other hand receive the recorded sensor data. The remote controlling station can select the type of sensor data need to be sent, either pressure or temperature and command the mote to switch off the unused sensor interface. In addition the base station can adapt the settings of the mote power management and sampling frequency based on the nature of the data received. Such feature has proven to be very advantageous for any smart system [11] to overcome uncertainties.

The base station is able to communicate with multiple motes. This can be accomplished by using a form of time-division multiplexing (TDM).

The data received from all motes at the base station are then sent to a PC via RS232 serial communication. Two general purposes PIC microcontrollers have been used in both sides of the systems. The MCU has internal analog to digital converter (ADC) and Universal Asynchronous Receiver Transmitter (UART) for testing and interfacing with a PC. The mote is operating using $3.3 \mathrm{~V}$ lithium battery that has a very high energy density and offers a longer battery life.

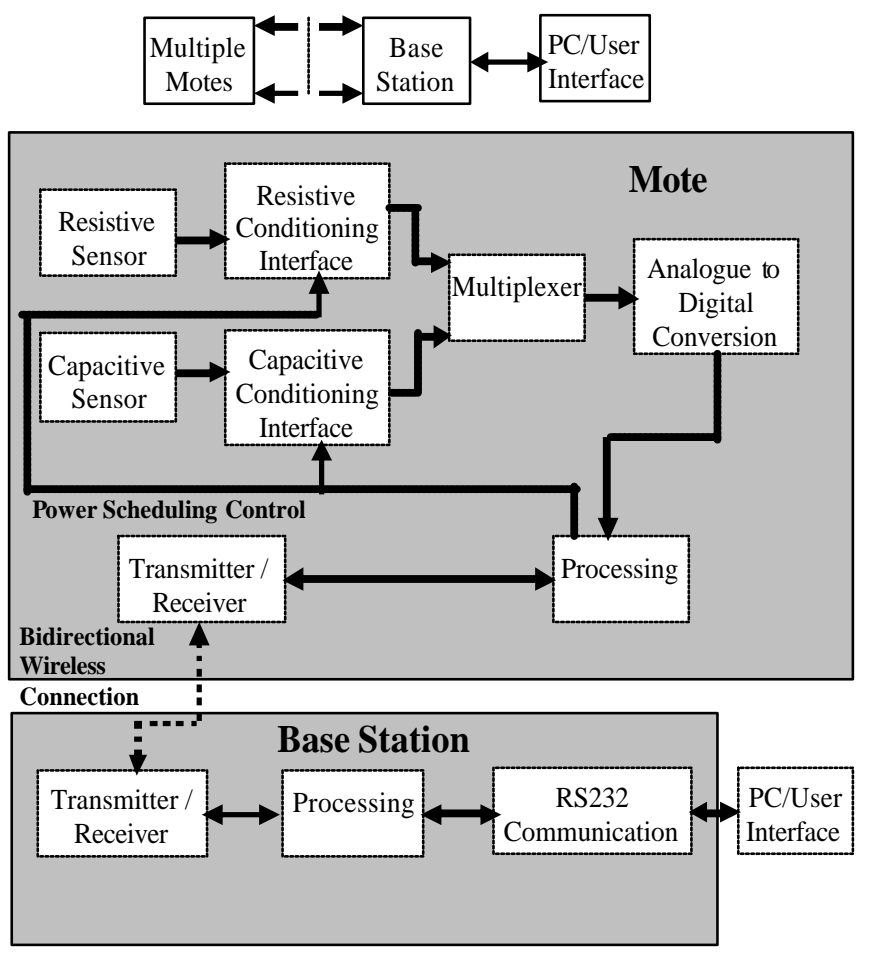

Figure 1. System overview

\section{SENSOR DEVELOPMENT AND MANUFACTURING}

PVDF is a low-density semi-crystalline material, consisting of long repeating chains of $-\mathrm{CF}_{2}-\mathrm{CH}_{2}-$ molecules. The crystalline region consists of a number of polymorphs, of which the $\alpha$ - and $\beta$-phase are most common. The $\beta$-phase is piezoelectric and has many advantages including its mechanical strength, wide dynamic range, flexibility and ease of fabrication $[12,13]$.

In a medical context, poled PVDF films have been popular in the development of planar pressure-measurement systems, where their flexibility and the ease with which electrode patterns can be attached has been a particular advantage. Micromachined devices using PVDF as a flexible element in the system have also been developed for use in an endoscopic grasper because of its high force sensitivity, large dynamic range and good linearity [14].

In this work, PVDF films were formed into a sandwich capacitor, which was then subjected to changing hydrostatic pressures. The films deformed under pressure and the resulting change in capacitance was transmitted wirelessly through the liquid to an external receiver.

\section{DESIGN OF THE SMART WIRELESS SYSTEM}

In this section, the design options for both resistive, capacitive signal conditioning circuits and power management are presented.

\section{A. Microcontrollers}

The family of Microchip PIC18Fx2 processors has many peripheral features that are useful in our application, while still drawing relatively small amounts of current (for example, approximately $5 \mathrm{~mA}$ at $20 \mathrm{MHz}$ ). The PIC18LF452 microcontroller (MCU) was selected for use on both the Mote and Base Station [15]. Some of the I/O features are common to both sides of the application, while others are specific to the unit.

\section{B. Transceivers}

The wireless aspect of the design was achieved using an offthe-shelf transceiver with $433 \mathrm{MHz}$ band using FSK (frequency shift keying) modulation. The ChipCon [16] transceiver plug and play module CC1000PP, as seen in Figure.2, is the optimised evaluation board for the single chip transceiver, CC1000. This chip features low power and completely configurable under the control of software for many frequency bands, signal strengths, and has support for frequency hopping.

The evaluation module is built with all the necessary passive components required for operation. The module is $28 \mathrm{~mm}$ by 20 $\mathrm{mm}$ with jumper terminals for fast prototyping. Therefore all external connections to the module are placed on the two jumper terminals. The supply voltage for the plug and play board is between 2.1 and $3.6 \mathrm{~V}$ and the filter is pre- mounted on the board. C. Wireless communication protocol

The wireless smart system relies on a prefabricated transceiver unit operated at frequency $433 \mathrm{MHz}$. Both FSK and Manchester encoding are employed by the unit at a maximum speed of $38.4 \mathrm{~Kb} / \mathrm{s}$. The transceiver is half-duplex, however switching between the two ways of transmission can be done quickly. Devices that are not actively transmitting should be either 
in receive or polling mode depending on the power restrictions of the device.

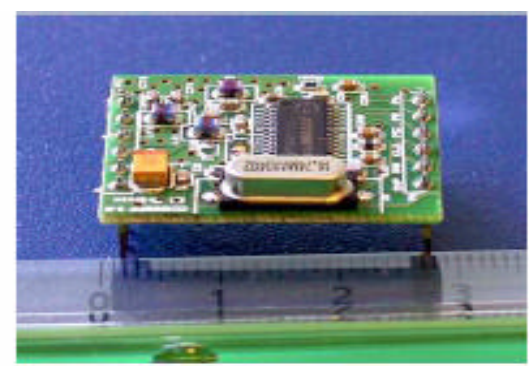

Figure 2. CC1000 Plug \& Play Evaluation Board

Several protocol standards were investigated as a possible alternative to designing a new protocol $[17,18]$. The standards found are mostly used in remote sensing and building/home automation and control. As a result of some concerns with using these standard protocols, it was decided that it would be best to develop a custom-written protocol.

The data link layer encapsulates the data blocks to be transmitted in a frame structure or a packet. The protocol developed for this purpose is bit-oriented where bytes are always transmitted with their most significant bit (MSB) first. The general frame structure is shown in Figure.3, with the size of each field. The command field is used to indicate the nature of the transmission. $\mathbf{I}$ is generally one byte, followed by variable or fixed-length data. The header check sum is 8-bit sum of the data packet bytes.

\begin{tabular}{|c|c|c|c|c|c|c|}
\hline $\begin{array}{c}\text { Start } \\
(1)\end{array}$ & $\begin{array}{c}\text { length } \\
(1)\end{array}$ & $\begin{array}{c}\text { Mote } \\
\text { address } \\
(2)\end{array}$ & $\begin{array}{c}\text { Base } \\
\text { address } \\
(1)\end{array}$ & $\begin{array}{c}\text { Command } \\
(1)\end{array}$ & $\begin{array}{c}\text { Date } \\
(1-250)\end{array}$ & $\begin{array}{c}\text { Checksum } \\
\text { (1) }\end{array}$ \\
\hline
\end{tabular}

Figure 3.General packet structure

D. Resistive interface

Anderson Loop [19] circuit topology has been selected for the resistive interface circuitry. In this configuration, a practical dual-differential subtractor with sufficiently high input impedance and rejection of unwanted signals has been introduced.

The schematic of the developed resistive circuitry is shown in Figure.4. For the constant current excitation, a voltage regulator is used to get a precision voltage drop $\mathrm{R}_{\text {bias. }}$. This creates the bias current for the current mirror, which is composed of two NPN Bipolar Junction Transistors (2N3904). In this way, the current entering the collector of Q1 is also induced at the collector of Q2. However, the current can vary for a particular transistor with temperature fluctuations. To overcome this, the transistors are joined together using epoxy to ensure getting stable current.

E. Capacitive interface

In Figure.4, the block diagram of the capacitive conditioning interface circuitry is given. A dual CMOS timer is used to convert the two capacitances, sensor and reference, to an output square signal with a variable frequency. Since the ADC in the next stage will only accept a single input, a switch (ADG836) is used to take a reference reading first and then the sensor reading. The reference will be used to calibrate the sensor readings. It was found that the circuit could measure a range of capacitance from $5 \mathrm{pF}$ to $500 \mathrm{pF}$. The TLC556 dual CMOS timer (manufactured by Texas Instrument [20]) is used in astable configuration to generate a square wave signal with approximately $50 \%$ duty cycle. The system was developed specifically for testing the performance of the capacitive sensors [21].

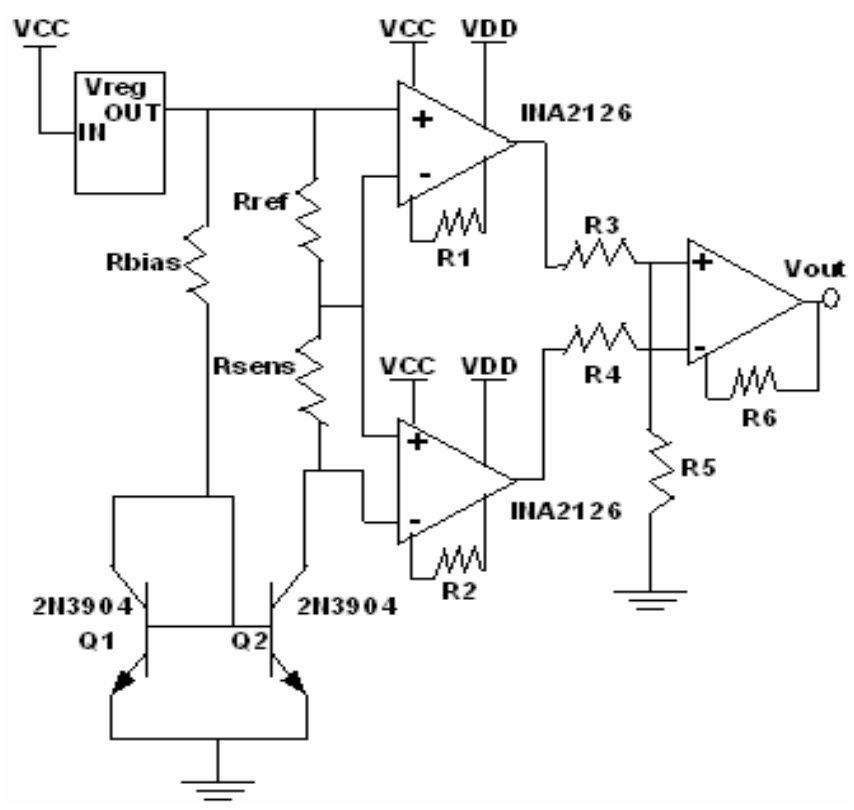

Figure 4.Resistive interface circuitry schematic

The CD4045 Phase locked Loop (PLL) unit is used to convert the frequency to voltage and use the MCU in the mote to convert it to digital information before sending it wirelessly. The PLL circuit was found to be more adequate and has a higher sensitivity for changes in the desired capacitance range. In addition the PLL circuit was found to have lower power consumption when compared to standard frequency-to-voltage converter configurations and other interface circuits as clarified in Table.1.

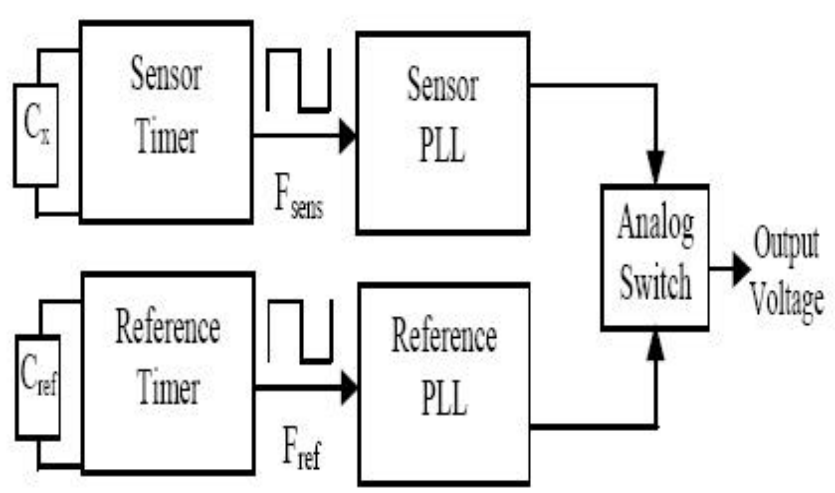

Figure 4. Block diagram of the capacitive circuitry in the mote system 


\section{SYSTEM POWER MANAGEMENT}

Since it is required to reduce power consumption on the mote as much as possible, Power scheduling has been designed carefully to control the different parts of the system. Figure. 5 shows the power flow mechanism which is mainly controlled by the processor unit. The transceiver and MCU have a "sleep" mode that can be controlled from the software.

Table 1. Power Consumption Compression for different capacitive sensor interface circuits

\begin{tabular}{|c|c|c|}
\hline Interface & $\begin{array}{c}\text { Current } \\
\text { Consumption }\end{array}$ & $\begin{array}{c}\text { Supply } \\
\text { Voltage } \\
\text { (minimum) }\end{array}$ \\
\hline $\begin{array}{c}\text { Universal Capacitive } \\
\text { Readout IC - MS3310 [35] }\end{array}$ & 2.9 to $6.0 \mathrm{~mA}$ & $5 \mathrm{~V}$ \\
\hline AC Bridge (simulated) & 2 to $10 \mathrm{~mA}$ & None \\
\hline $\begin{array}{c}\text { Anderson Loop [34] } \\
\text { (Implemented) }\end{array}$ & 1 to $3 \mathrm{~mA}$ & $3 \mathrm{~V}$ \\
\hline $\begin{array}{c}\text { C-F-V, (using F-V converter) } \\
\text { LM2907 (Implemented) }\end{array}$ & 5 to $8 \mathrm{~mA}$ & $3 \mathrm{~V}$ \\
\hline $\begin{array}{c}\text { C-F-V, (using F-V converter) } \\
\text { TC9400 (Implemented) }\end{array}$ & 1.5 to $6 \mathrm{~mA}$ & $10 \mathrm{~V}$ \\
\hline C-F-V, PLL (Implemented) & $<500 \mu \mathrm{A}$ & $2.3 \mathrm{~V}$ \\
\hline
\end{tabular}

As shown above, the sensor signal conditioning circuits can be switched on or off by the control signals of the processor unit. A CMOS analog switch is used for this purpose. Table. 2 clarifies the power scheduling options for the sensor interface circuits. In order to preserve the power, certain modules within the mote system are powered off when they are not in use.

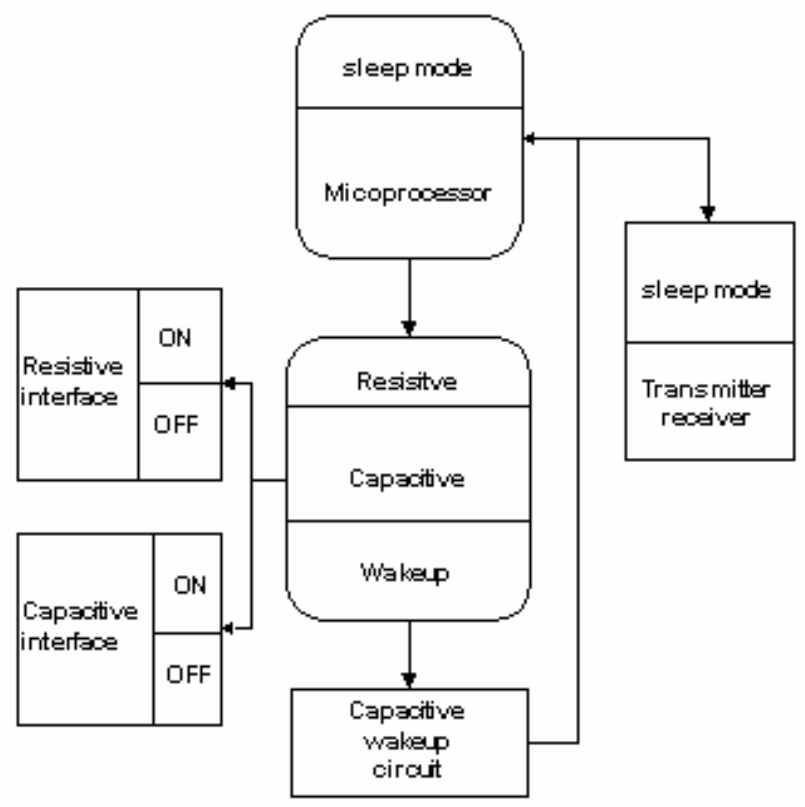

Figure 5. Power comrol of the mote system
Table 2. Sensors interfaces power scheduling

\begin{tabular}{|c|c|c|c|}
\hline$\mu \mathbf{P}$ & RESISTIVE & $\begin{array}{c}\text { CAPACITIV } \\
\mathbf{E}\end{array}$ & $\begin{array}{c}\text { WAKEU } \\
\mathbf{P}\end{array}$ \\
\hline Wakeup & ON & OFF & OFF \\
\hline Wakeup & OFF & ON & OFF \\
\hline Sleep & OFF & OFF & ON \\
\hline
\end{tabular}

At any one time, mote system is configured to read either resistive or capacitive samples. For the resistive interface, mote is powering on/off for each sample (max sampling rate $200 \mathrm{sps}$ ). The capacitive interface is powered from a $2 \mathrm{~V}$ supply which is less than that for the resistive and has a large start-up time of about $200 \mathrm{~ms}$. On the other hand, resistive interface consumes high current $(15 \mathrm{~mA})$ and has a low start-up time $\quad(\sim 1 \mathrm{~ms})$. This makes it infeasible to be turned on/off the capacitive unit for each sample since it draws already a very low current $(\sim 400 \mu \mathrm{A})$. As a result, the capacitive interface will be left on constantly when being used.

For further power consumption reduction, a wakeup circuit, which allows the processor to enter sleep mode while capacitive readings are not changing, has been developed. It has been noticed that wakeup circuit is not suited for the resistive interface where a higher power is needed to keep the circuit operating constantly

\section{A. Wakeup circuit design}

The purpose of the wakeup circuit is to put the MCU in sleep mode when the input signal is not changing. When the signal starts to give a significant change, an interrupt is generated that wakes up the MCU and it will start sampling again. The circuit is designed as shown in Figure.6.

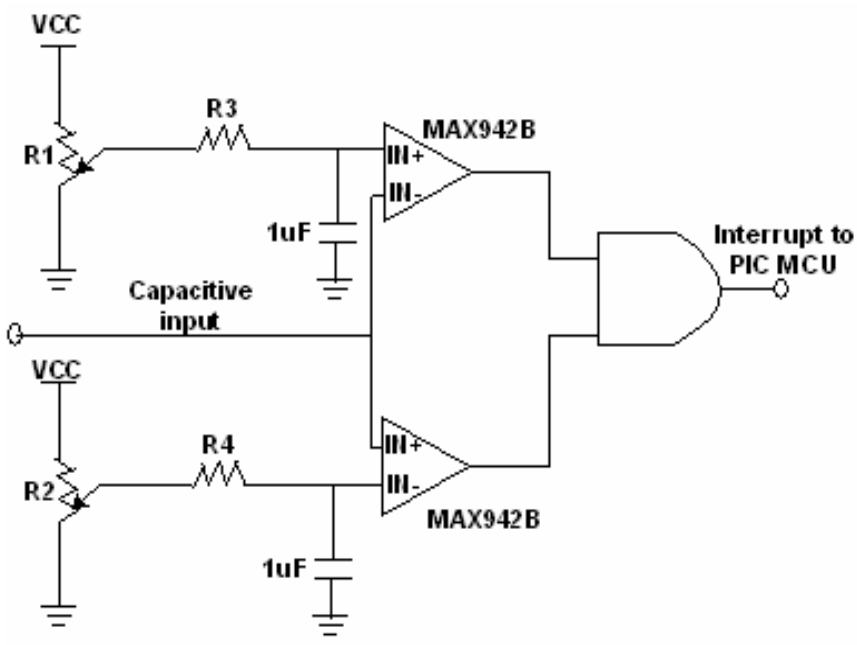

Figure 6. Wakeup circuit diagram

The design is based on the principle of window detector, where two digitally controlled potentiometers are used to set the upper and lower trip points of the two comparators. At the beginning of operation, the MCU decides when the capacitive samples are not changing, and then it will set the upper and lower limits of the 
window and powers up the wakeup circuit. The MCU will be in sleep mode when the sensor goes outside the limit. Then an interrupt signal will be generated to wakeup the MCU that will power down the wakeup circuit to save power.

The above design draws bout $700 \mu \mathrm{A}$ and the interrupt signal will be generated by a NAND gate that outputs zero when the sensor is within the window range. If the sensor goes above the uppertrip or below the lower trip, the desired output is high, that will interrupt the MCU from the sleep mode.

\section{B. Power usage}

Table.4 summarizes the current consumptions of the different mote system units and their working duty cycles. It is clear from the table that most of the power is consumed by the sensors signal conditioning circuits and the transceiver. It can be noticed that the wakeup circuit draws higher current $(\sim 0.7 \mathrm{~mA})$ when it is ON than the capacitive interface when it is working. However the capacitive with the wakeup circuit still draw less current than the MCU. The maximum efficiency can be reached with the capacitive when the signal doesn't change considerably over time.

Table 4. Current consumption specifications of the mote system

\begin{tabular}{|c|c|c|c|}
\hline MOTE PART & $\begin{array}{c}\text { CURRENT } \\
\text { VALUE }\end{array}$ & UNITS & $\begin{array}{c}\text { DUTY } \\
\text { CYCLE }\end{array}$ \\
\hline $\begin{array}{c}\text { Transceiver sleep } \\
\text { mode }\end{array}$ & 0.5 & $\mu \mathrm{A}$ & \\
\hline $\begin{array}{c}\text { Transmitter current } \\
10(\mathrm{at} \\
10 \mathrm{dBm})\end{array}$ & $\mathrm{mA}$ & $6.56 \%$ \\
\hline Receiver & 8 & $\mathrm{~mA}$ & $5 \%$ \\
\hline Resistive circuitry & 1.5 & $\mathrm{~mA}$ & $20 \%$ \\
\hline Capacitive circuitry & 400 & $\mu \mathrm{A}$ & $100 \%$ \\
\hline Wakeup circuit & 0.7 & $\mathrm{~mA}$ & $100 \%$ \\
\hline MCU current & 3 & $\mathrm{~mA}$ & $100 \%$ \\
\hline MCU sleep current & 16 & $\mu \mathrm{A}$ & \\
\hline
\end{tabular}

\section{TESTING SYSTEM PERFORMANCE}

To examine the performance of the overall wireless system, a test bench has been developed for this purpose. It is consisted of a tube that has been filled with water. The mote unit will be placed in a small waterproof box attached to certain weight that pushes it down to the bottom of the tube. Such movement causes a uniform pressure changes determined by the height which has been calculated in prior to cover the required pressure range.

A commercial temperature sensor from ANDIGILOG [22] has been used for this purpose to examine the performance of the resistive interface circuitry. The two sensors are exposed to the water through two holes drilled on the top of the water proof box and covered by a flexible material to prevent water leak. The amount of pressure attenuation caused by this material has been calculated in order to be tolerated later on.

Figure.7 shows the response of the PVDF sensors to pressure in the range $0-15 \mathrm{kPa}$. The operation of the PLL unit causes a nonlinear response, however it can be seen that the voltage changed with pressure over the entire range.

An external heating source has been used to change the water temperature from 0 to $80{ }^{\circ} \mathrm{C}$. The thermal characteristic of the temperature sensor is shown in Figure.8. The characterization of the temperature sensors indicated a maximum sensitivity of 20 $\mathrm{mV} /{ }^{\circ} \mathrm{C}$ with a base voltage of $0.1 \mathrm{~V}$. Obviously the resistive circuit performs well during the test range and configured to be used with any other sensor based on setting the reference, bias and gain resistors.

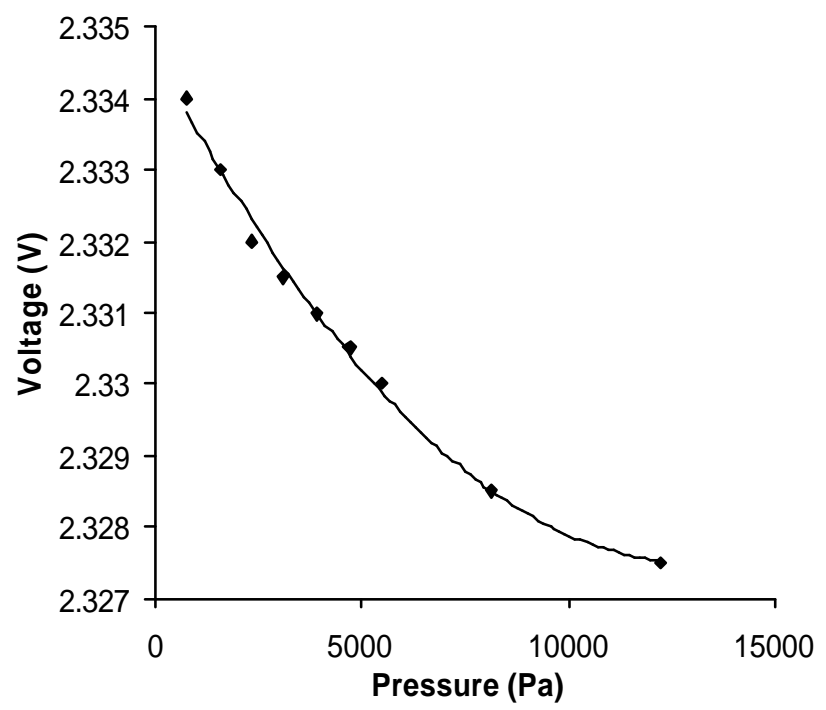

Figure 7. Output voltage change with pressure using the PVDF sensor

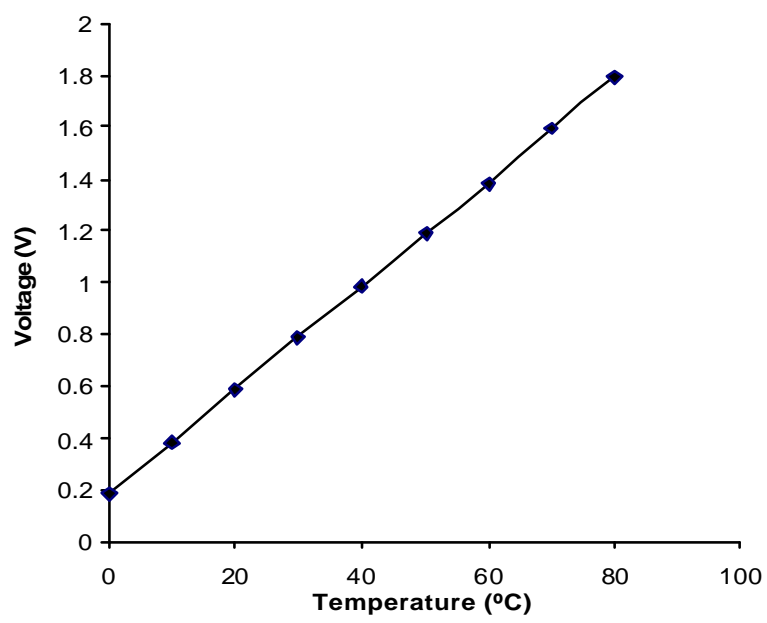

Figure 8.Temperature sensitivity of the temperature sensor 


\section{CONCLUSIONS}

This paper describes the design and implementation of wireless system suitable for environmental monitoring. Measuring the water pressure and temperature signals was the main scope of the work. The system reads data from two types of sensors, resistive (temperature) and capacitive (pressure). Anderson circuit configuration has been used efficiently for the resistive sensor, since it offers linear like behavior. New features have been added to the design to improve the stability of the excitation current and make the circuit configurable for different sensor values. The capacitive interface is based on capacitance-frequency-voltage conversion that uses PLL to convert the frequency to voltage. The PLL unit has $1 \%$ linearity and consumes a low power.

The pressure sensing properties of sandwich capacitors based on PVDF were evaluated using the specially constructed wireless data acquisition system for the capacitive interface. It was seen that each material displayed a high sensitivity to pressure changes in the range $0-15 \mathrm{kPa}$. It can be concluded that PVDF sensors could find applications in systems where pressures measurements over a large range are required. Two mechanisms have been adopted to reduce further the overall power consumption. These are power scheduling and wakeup circuit for the capacitive interface. The first one is implemented fully by the MCU in order to switch on/off all the system units. A wakeup circuit has been designed to interrupt the MCU when it is in the sleep mode and the capacitive samples are changing significantly. It was found that such design is more suited for the capacitive interface than the resistive one where the circuit consumes much less power even if it is kept working continuously.

\section{A. ACKNOWLEDGMENTS}

This work was supported by the Enterprise Ireland Commercialisation Fund 2003, under technology development phase, as part of the MIAPS project, reference no. CFTD/03/425.

\section{B. REFERENCES}

[1] T. Akin, K. Najafi, and R. Bradley, "A wireless implantable multichannel digital neural recording system for a micromachined sieve electrode," IEEE Journal of solid-state circuits, vol. 33, 1998, pp. 109- 118.

[2] E. Johannessen, L. Wang, C. Wyse, D. Cumming, and J. Cooper, "Biocompatibility of a Lab-on-a-Pill Sensor in Artificial Gastrointestinal Environments", IEEE Biomedical Engineering, vol 53, 2006, pp.2333-2340.

[3] C. A. Grimes, M. K. Jain, R. S. Singh, Q. Cai, A. Mason, K. Takahata, Y. Gianchandani, "Magnetoelastic microsensors for environmental monitoring," Proc 14thIEEE conf. Micro electro mechanical systems, 2000, pp.278-281.

[4] S. Bellis, K. Delaney, B. O’Flynn, J. Barton, K. Razeeb, and C. O'Mathuna, "Development of filed programmable modular wireless sensor network nodes for ambient systems," Computer Commutations, vol 28, 2005, pp.15311544.

[5] Y. K. Seok, G. Joonho, K. Jinbong, K. Hong-Jeong, K. Kyunghyun, P. Daesik, K. Myeung su, S. Hyungcheol, L. Kwyro, K. Juhyoun, Y. Euisik, “A miniaturized low-power wireless remote environmental monitoring system based on electrochemical analysis," Sensors and Actuators, vol. 102, 2004, pp. 27-34.

[6] A.DeHannis, and K. Wise, "A wireless microsystem for the remote sensing of pressure, temperature and reative humidity" IEEE Microelectromechanical Systems, vol 14, 2005, pp.12-22.

[7] A. Mohan, A.P. Malshe, S. Aravamudhan, and S.Bhansali, "Piezoresistive MEMS pressure sensor and packaging for harsh oceanic environment," Proc 54th conf. IEEE Electronic Components and Technology Conference, vol1, 2004, pp.948-950.

[8] S. Anumalla, B. Ramamurthy, D.C. Gosselin, and M. Burbach, "Ground water monitoring using smart sensors," IEEE Conf. Electro Information Technology, 2005.

[9] E. Johannessen, W. Lei, C. Li, T. Tong Boon, A. Mansour, A. Alexander, R. Stuart W. J, Y. Philippa S, M. Alan F, F. Brian W, B. Steve P, C. David R. S, and C. Jonathan M, "Implementation of multichannel sensors for remote biomedical measurements in a Microsystems format," IEEE Trans. Biomedical Engineering, vol. 51, 2004, pp. 525-523.

[10] K. Arshak, and E. Jafer, "Design of low power smart wireless system for Multi-syatem monitoring," Proc IEEE Conf. Sensors, CA (USA), Oct 2005.

[11] R. Puers, "Sensor, sensor interfacing and front-end data management for stand-alone Microsystems," J. Micromech. Microeng, vol..9, 1999.

[12] P.A. Payne and Q.X. Chen, "Polymer materials for pressure measurement," Physiological Pressure Measurement, IEE Colloquium on (1990) 7/1.

[13] Measurement Specialities, Piezo Film Sensors: Technical Manual, Norristown, 1999, p. 86.

[14] J. Dargahi, M. Parameswaran and S. Payandeh, "A Micromachined Piezoelectrk Tactile Sensor for use in Endoscopic Graspers," Int Conference on Intelligent Robots and Systems, Victoria, B.C., Canada, 1998.

[15] PIC family Microcontrollers: www.microchip.com.

[16] CC1000 Chipcpn RF Transceiver data sheet: http://www.chipcon.com.

[17] M. Sgroi, J.L. da Silva, F. De Bernardinis, F. Burghardt, A. Sangiovanni-Vincentelli, and J.Rabaey, "Designing wireless protocols: methodology and applications", IEEE Proc. ICASSP, vol.6, 2000, pp. 3726 - 3729. 
[18] W. B.Heinzelman, A.P.Chandrakasan, and H.Balakrishnan, "An application-specific protocol architecture for wireless microsensor networks", IEEE Trans Wireless communications, vol.1, 2002, pp. $660-670$.

[19] Anderson, K.F, “ NASA's Anderson loop,” IEEE Instrumentation and Measurement magazine, vol. 1, 1998, pp. 5-15.
[20] TLC556 Dual CMOS Timer, manual sheet, Texas Instruments: http://focus.ti.com/docs/prod/folders/print/tlc556.html.

[21] A. Arshak, K. Arshak, D. Morris, O. Korostynska and E. Jafer, "Investigation of TiO2 thick film capacitors for use as strain gauge sensors," Sensors and Actuators, vol 122, pp.242-249.

[22] ASM121 Ultra Low Power Temperature Sensor, data sheet: http://www.andigilog.com/. 